Journal of Mathematics and Statistics 5 (3):167-170, 2009

ISSN 1549-3644

(C) 2009 Science Publications

\title{
A New Block Method for Special Third Order Ordinary Differential Equations
}

\author{
B.T. Olabode and Y. Yusuph \\ Department of Mathematical Sciences, \\ Federal University of Technology, Akure, Nigeria \\ Department of Mathematics and Computer Science, \\ Federal University of Technology, Minna, Nigeria
}

\begin{abstract}
A linear multistep method for the direct solution of initial value problems of ordinary differential equations was presented in this article. Collocation approximation method was adopted in the derivation of the scheme and then the scheme was applied as simultaneous integrator to special third order initial value problem of ordinary differential equations. The new block method possessed the desirable feature of Runge-Kutta method of being self-starting and eliminated the use of predictors. The 3-step block method is P-stable, consistent and more accurate than the existing one. Experimental results confirmed the superiority of the new scheme over the existing method.
\end{abstract}

Key words: Linear multistep method, P-stable, third order IVPS of ODES, Interval of periodicity, corrector and predictor

\section{INTRODUCTION}

The mathematical formulation of physical phenomena in science and engineering often leads to initial value problems of the form:

$y^{\prime \prime \prime}=f(x, y), y(a)=y_{0} y^{\prime}(a) \eta_{0}, y^{\prime \prime}(a)=\eta_{1}$

However, only a limited number of analytical methods are available for solving (1) directly without reducing to a first order system of initial value problems. Some authors have proposed solution to higher order initial value problems of ordinary differential equations using different approaches ${ }^{[1-5]}$. In particular Awoyemi and $\operatorname{Idowu}^{[2]}$ developed a class hybrid collocation method for third order of ordinary differential equations. Awoyemi ${ }^{[1]}$ derived a p-stable linear multistep method for general third order initial value problems of ordinary differential equations which is to be used in form of predictor-corrector forms and like most linear multistep methods, they require starting values from Runge-Kutta methods or any other onestep methods. The predictors are also developed in the same way as correctors. Moreover, the block methods in Fatunla ${ }^{[3]}$ are in form of discrete and are proposed for non-stiff special second order ordinary differential equations in form of a predictor- corrector integration process. Also like other linear multistep methods are usually applied to the initial value problems as a single formula but they are not self-starting; and they advance the numerical integration of the ordinary differential equations in one-step at a time, which leads to overlapping of the piecewise polynomials solution model.

There is the need to develop a method which is self-starting, eliminating the use of predictors with better accuracy and efficiency. This study, therefore propose a block multistep method for the direct solution of third order initial value problems of ordinary differential equations.

\section{MATERIAL AND METHOD}

A power series of a single variable $\mathrm{x}$ in the form:

$P(x)=\sum_{j=0}^{\infty} a_{j} x^{j}$

is used as the basis or trial function, to produce the approximate solution as:

$$
y(x)=\sum_{j=0}^{k+2} a_{j} x^{j} \quad a_{j} \in R, j=0(1) k+2, y \in C^{m}(a, b) \subset P(x)
$$

Assuming an approximate solution to (1) in the form of (3) whose high derivatives are:

$$
y^{\prime}(x)=\sum_{j=0}^{k+2} j(j-1) a_{j} x^{j-1}
$$




$$
\begin{aligned}
& y^{\prime \prime}(x)=\sum_{j=0}^{k+2} j(j-1)(j-2) a_{j} x^{j-2} \\
& y^{\prime \prime \prime}(x)=\sum_{j=0}^{k+2} j(j-1)(j-2)(j-3) a_{j} x^{j-3}
\end{aligned}
$$

From Eq. (2) and (6)

$$
\sum_{j=0}^{k+2} j(j-1)(j-2)(j-3) a_{j} x^{j-3}=f(x, y)
$$

where, $a_{j}$ is the parameters to be determined.

Collocating (7) at the mesh-points $X=x_{n+j}$, $\mathrm{j}=0(1) \mathrm{k}$ and interpolating (3) at $\mathrm{x}=\mathrm{x}_{\mathrm{n}+\mathrm{j}}, \mathrm{j}=0(2) \mathrm{k}-1$.

Putting in the matrix equation form and then solved to obtain the values of parameters $a_{j}$ 's, $j=0,1, .$. which is substituted in (3), after some manipulation, yields the new continuous method expressed in the form:

$$
y(x)=\sum_{j=0}^{k-1} a_{j}(x) y_{n+j}+\sum_{j=0}^{k} \beta_{j}(x) f_{n+j}
$$

Derivation of first-block of 3-step method: The firstblock of 3-step method for the solution of (1) can be expressed by the following matrix difference equation.

$A^{(0)} \cdot y_{q}=A^{(1)} \cdot y_{q-1}+h^{3} B^{(0)} \cdot F_{q}+B F_{q-1}$

And the coefficients $\alpha_{j}{ }^{\prime}$ s and $\beta_{j}$ 's of the continuous scheme (8) are thus:

$$
\begin{aligned}
& t \quad=\frac{x-x_{n+1}}{h} \\
& \alpha_{0}(t)=\frac{1}{2}\left\{t^{2}-t\right\} \\
& \alpha_{1}(t)=-\left\{t^{2}-1\right\} \\
& \alpha_{2}(t)=\frac{1}{2}\left\{t^{2}+t\right. \\
& \beta_{0}(t)=\frac{h^{3}}{720}\left\{-t^{6}+6 t^{5}-10 t^{4}+11 t^{2}-6 t\right\} \\
& \beta_{1}(t)=\frac{h^{3}}{720}\left\{3 t^{6}-12 t^{5}-15 t^{4}+120 t^{3}+12 t^{2}-108 t\right. \\
& \beta_{2}(t)=\frac{h^{3}}{720}\left\{-3 t^{6}+6 t^{5}+30 t^{4}-27 t^{2}-6 t\right\} \\
& \beta_{3}(t)=\frac{h^{3}}{720}\left\{t^{6}-5 t^{4}+4 t^{2}\right\}
\end{aligned}
$$

Evaluating (10) at $\mathrm{x}=\mathrm{x}_{\mathrm{n}+3}$ yield the discrete scheme: $\mathrm{y}_{\mathrm{n}+3}-3 \mathrm{y}_{\mathrm{n}+2}+3 \mathrm{y}_{\mathrm{n}+1}-\mathrm{y}_{\mathrm{n}}=\frac{\mathrm{h}^{3}}{2}\left\{\mathrm{f}_{\mathrm{n}+2}+\mathrm{f}_{\mathrm{n}+1}\right\}$

It is $\mathrm{P}$-stable. The order $\mathrm{P}=5$ i.e., $\mathrm{C}_{\mathrm{p}+2}=1 / 240$.

The first derivatives of (10) are, noting that:

$$
\begin{aligned}
& \frac{\mathrm{dt}}{\mathrm{dx}}=\frac{1}{\mathrm{~h}} \text { and } \frac{\mathrm{d}^{2} \mathrm{t}}{\mathrm{dx}^{2}}=\frac{1}{\mathrm{~h}^{2}} \\
& \alpha_{0}^{\prime}=\frac{1}{2 \mathrm{~h}}\{2 \mathrm{t}-1\} \\
& \alpha_{1}^{\prime}=-2 \mathrm{t} / \mathrm{h} \\
& \alpha_{2}^{\prime}=\frac{1}{2 \mathrm{~h}}\{2 \mathrm{t}+1\} \\
& \beta_{0}^{\prime}=\frac{\mathrm{h}^{2}}{720}\left\{6 \mathrm{t}^{5}+30 \mathrm{t}^{4}-40 \mathrm{t}^{3}+22 \mathrm{t}-6\right\} \\
& \beta_{1}^{\prime}=\frac{\mathrm{h}^{2}}{720}\left\{18 \mathrm{t}^{5}-60 \mathrm{t}^{4}-60 \mathrm{t}^{3}+360 \mathrm{t}^{2}+24 \mathrm{t}-108\right\} \\
& \beta_{2}^{\prime}=\frac{\mathrm{h}^{2}}{720}\left\{-18 \mathrm{t}^{5}+30 \mathrm{t}^{4}+120 \mathrm{t}^{3}-54 \mathrm{t}-6\right\} \\
& \beta_{3}^{\prime}=\frac{\mathrm{h}^{2}}{720}\left\{6 \mathrm{t}^{5}-20 \mathrm{t}^{3}+8 \mathrm{t}\right\}
\end{aligned}
$$

And the second derivatives are:

$$
\begin{aligned}
& \alpha_{0}^{\prime \prime}(\mathrm{t})=\frac{1}{\mathrm{~h}^{2}} \\
& \alpha_{1}^{\prime \prime}(\mathrm{t})=\frac{-2}{\mathrm{~h}^{2}} \\
& \alpha_{2}^{\prime \prime}(\mathrm{t})=\frac{1}{\mathrm{~h}^{2}} \\
& \beta_{0}^{\prime \prime}=\frac{\mathrm{h}}{720}\left\{-30 \mathrm{t}^{4}+120 \mathrm{t}^{3}-120 \mathrm{t}^{2}+22\right\} \\
& \beta_{1}^{\prime \prime}(\mathrm{t})=\frac{\mathrm{h}}{720}\left\{90 \mathrm{t}^{4}-240 \mathrm{t}^{3}-180 \mathrm{t}^{2}+720 \mathrm{t}+24\right\} \\
& \beta_{2}^{\prime \prime}=\frac{\mathrm{h}}{720}\left\{-90 \mathrm{t}^{4}+120 \mathrm{t}^{3}+360 \mathrm{t}^{2}-54\right\} \\
& \beta_{3}^{\prime \prime}=\frac{\mathrm{h}}{720}\left\{30 \mathrm{t}^{4}-60 \mathrm{t}^{2}+8\right\}
\end{aligned}
$$

Special application of 3-step method: Evaluating (10) at $\mathrm{x}=\mathrm{x}_{\mathrm{n}+3}$ yield respectively (11):

$$
y_{n+3}-3 y_{n+2}+3 y_{n+1}-y_{n}=\frac{h^{3}}{2}\left\{f_{n+2}+f_{n+1}\right\}
$$

Improving the block method by considering additional equation arising from the first and second derivative functions: 


$$
\begin{aligned}
& \frac{d u(x)}{d x}=Z(x), \frac{d u(a)}{d x}=Z_{0} \\
& \frac{d^{2} u(x)}{d x^{2}}=Z^{\prime}(x), \frac{d^{2} u(a)}{d x^{2}}=Z_{0}^{\prime}
\end{aligned}
$$

and continuity equation imposed at $\mathrm{x}=\mathrm{x}_{\mathrm{n}+3}$ of the form:

$\mathrm{Z}\left(\mathrm{x}_{\mathrm{n}+3}\right) \mathrm{x}_{\mathrm{n}} \leq \mathrm{x} \leq \mathrm{x}_{\mathrm{n}+3}=\mathrm{Z}\left(\mathrm{x}_{\mathrm{n}+3}\right) \mathrm{x}_{\mathrm{n}+3} \leq \mathrm{x} \leq \mathrm{x}_{\mathrm{n}+3}$

Using the first and second conditions in (1) on (14) to obtain:

$$
\begin{aligned}
& 2 h Z_{0}+y_{n+2}+4 y_{n+1}+3 y_{n}=\frac{h^{3}}{720}\left\{\begin{array}{l}
12 f_{n+3}-48 f_{n+2} \\
+420 f_{n+1}+96 f_{n}
\end{array}\right\} \\
& h^{2} Z_{0}^{\prime}-y_{n+2}+2 y_{n+1}-y_{n}=\frac{h^{3}}{720}\left\{\begin{array}{l}
-22 f_{n+3}+96 f_{n+2} \\
-546 f_{n+1}-248 f_{n}
\end{array}\right\}
\end{aligned}
$$

Combining (11) and (16) yield the first block and solving simultaneously to start the integration process. There are two options to move the integration process forward after the first sub-interval. First, we could use the block method (the combination of (11) and (16) in its present form i.e., block method over sub-intervals that do over-lap $\left(\mathrm{x}_{\mathrm{o}}, \mathrm{x}_{3}\right\},\left\{\mathrm{x}_{1}, \mathrm{x}_{4}\right\} \ldots,\left\{\mathrm{x}_{\mathrm{N}-3}, \mathrm{x}_{\mathrm{N}}\right\}$. The second options is to proceed by explicitly obtaining initial conditions at $x_{n+3}, n=0,3 \ldots N-3$, using the computed values $\mathrm{U}\left(\mathrm{x}_{\mathrm{n}+3}\right)=\mathrm{y}_{\mathrm{n}+3}$ and $\mathrm{Z}\left(\mathrm{x}_{\mathrm{n}+3}\right)=\mathrm{Z}_{\mathrm{n}+3}$ over sub-intervals $\left(\mathrm{x}_{0}, \mathrm{x}_{3}\right\}\left\{\mathrm{x}_{3}, \mathrm{x}_{6}\right\}, \ldots\left\{\mathrm{x}_{\mathrm{N}-3}, \mathrm{x}_{\mathrm{N}}\right\}$ which do not over-lap.

\section{RESULTS AND DISCUSSION}

Numerical experiments and results: This section deals with the implementation of the algorithm proposed for the initial value problems.

\section{Problem 1:}

$$
\mathrm{y}^{\prime \prime \prime}=-\mathrm{y}
$$$$
y(0)=1, \quad y^{\prime}(0)=-1, \quad y^{\prime \prime}(0)=1
$$

Theoretical solution is:

$$
y(x)=e^{-x}
$$

\section{Problem 2:}

$$
\begin{aligned}
& y^{\prime \prime \prime}=3 \sin x \\
& y(0)=1, \quad y^{\prime}(0)=0, \quad y^{\prime \prime}(0)=-2
\end{aligned}
$$

Theoretical solution is:

$$
y(x)=3 \cos x+\frac{x^{2}}{2}-2
$$

Problem 3:

$$
\begin{aligned}
& y^{\prime \prime \prime}=e^{x} \\
& y(0)=3, \quad y^{\prime}(0)=1, \quad y^{\prime \prime}(0)=5
\end{aligned}
$$

Theoretical solution is:

$$
y(x)=2+2 x^{2}+e^{x}
$$

\section{Problem 4:}

$y^{\prime \prime \prime}=-e^{x}$

$y(0)=1, \quad y^{\prime}(0)=-1, \quad y^{\prime \prime}(0)=3$

Theoretical solution is:

$y(x)=2+2 x^{2}-e^{x}$

In Table 1, the 3-Step Block Method has maximum absolute error of $6.3596 \mathrm{E}-10$ while the maximum absolute error of Awoyemi ${ }^{[1]}$ is $1.36929 \mathrm{E}-09$. Both

\begin{tabular}{|c|c|c|c|c|}
\hline$X$ & $\begin{array}{l}\text { Exact } \\
\text { solution } \\
\mathrm{y}(\mathrm{x})\end{array}$ & $\begin{array}{l}\text { 3-step block } \\
\text { method } \\
\text { y-computed }\end{array}$ & $\begin{array}{l}\text { Error in } \\
\text { 3-step } \\
\text { block method }\end{array}$ & $\begin{array}{l}\text { Awoyemi }{ }^{[1]} \\
\mathrm{k}=3 \text { method }\end{array}$ \\
\hline 0.1 & 0.90483742 & 904837417 & $3596 \mathrm{E}-11$ & $1.36929 \mathrm{E}$ \\
\hline 0.2 & 0 & & & \\
\hline 0.3 & 0.740 & & 99 & -07 \\
\hline 0.4 & & & & \\
\hline 0.5 & & & & \\
\hline 0.6 & & & & \\
\hline 0.7 & & & & \\
\hline 0.8 & & & & \\
\hline 0.9 & & & & 41E-06 \\
\hline 1.0 & 0 & & & $5.14755 \mathrm{E}-06$ \\
\hline 1.1 & 33287108 & & $2.90019 \mathrm{E}$ & $6.74582 \mathrm{E}-06$ \\
\hline 1.2 & 0.30119421 & 0.301194258 & 4.60878E-08 & 8.60677E-06 \\
\hline
\end{tabular}
methods are of the same order and step but block method is seen to be more accurate than Awoyemi ${ }^{[1]}$.

In Table 2-4, the maximum absolute errors for problems 2,3 and 4 are observed to be $1.65922 \mathrm{E}-10$, $7.56479 \mathrm{E}-11$ and $7.24352 \mathrm{E}-10$, respectively. The lower values of these errors showed that the new method is accurate.

Table 1: Problem 1: Accuracy comparison of the new block method and predictor-corrector method of Awoyemi ${ }^{[1]}$

Comparison of block method and predictor-corrector method for $\mathrm{h}=0.1$ 
Table 2: Problem 2: Absolute errors

\begin{tabular}{llll}
\hline \multicolumn{3}{c}{ Comparison of theoretical/approximate solution, $\mathrm{h}=0.1$} \\
$-\mathrm{y}$ & $\begin{array}{l}\text { Exact } \\
\mathrm{X}\end{array}$ & solution $\mathrm{y}(\mathrm{x})$ & 3-step block method \\
\hline 0.1 & 0.990012496 & 0.990012496 & $1.65922 \mathrm{E}-10$ \\
0.2 & 0.960199734 & 0.960199734 & $4.76275 \mathrm{E}-10$ \\
0.3 & 0.911009467 & 0.911009468 & $6.23182 \mathrm{E}-10$ \\
0.4 & 0.843182982 & 0.843182982 & $2.91345 \mathrm{E}-10$ \\
0.5 & 0.757747686 & 0.757747685 & $8.71118 \mathrm{E}-10$ \\
0.6 & 0.656006845 & 0.656006841 & $3.92904 \mathrm{E}-09$ \\
0.7 & 0.539526562 & 0.539526552 & $9.55347 \mathrm{E}-09$ \\
0.8 & 0.410120128 & 0.410120110 & $1.80415 \mathrm{E}-08$ \\
0.9 & 0.269829905 & 0.269829875 & $3.03120 \mathrm{E}-08$ \\
1.0 & 0.120906918 & 0.120906870 & $4.73044 \mathrm{E}-08$ \\
1.1 & 0.034211636 & -0.034211706 & $7.00367 \mathrm{E}-08$ \\
1.2 & 0.192926737 & -0.192926836 & $9.96300 \mathrm{E}-08$ \\
\hline
\end{tabular}

Table 3: Problem 3: Absolute errors

Comparison of theoretical/approximate solution, $\mathrm{h}=0.1$

\begin{tabular}{llll} 
& & \multicolumn{2}{l}{ 3-step block method } \\
$\mathrm{X}$ & Exact solution $\mathrm{y}(\mathrm{x})$ & $\mathrm{y}$-computed & Errors \\
\hline 0.1 & 3.125170918 & 3.125170918 & $-7.56479 \mathrm{E}-11$ \\
0.2 & 3.301402758 & 3.301402760 & $1.83983 \mathrm{E}-09$ \\
0.3 & 3.529858808 & 3.529858812 & $4.42400 \mathrm{E}-09$ \\
0.4 & 3.811824698 & 3.811824708 & $1.03587 \mathrm{E}-08$ \\
0.5 & 4.148721271 & 4.148721282 & $1.12999 \mathrm{E}-08$ \\
0.6 & 4.542118800 & 4.542118815 & $1.46095 \mathrm{E}-08$ \\
0.7 & 4.993752707 & 4.993752728 & $2.05295 \mathrm{E}-08$ \\
0.8 & 5.505540928 & 5.505540948 & $1.95075 \mathrm{E}-08$ \\
0.9 & 6.079603111 & 6.079603122 & $1.08431 \mathrm{E}-08$ \\
1.0 & 6.718281828 & 6.718281830 & $0.154095 \mathrm{E}-08$ \\
1.1 & 7.424166024 & 7.424166010 & $1.39464 \mathrm{E}-08$ \\
1.2 & 8.200116923 & 8.200116886 & $3.67365 \mathrm{E}-08$ \\
\hline
\end{tabular}

Table 4: Problem 4: Absolute errors

\begin{tabular}{llll}
\hline \multicolumn{3}{c}{ Comparison of theoretical/approximate solution, $\mathrm{h}=0.1$} \\
$\mathrm{y}$ & $\begin{array}{l}\text { Exact } \\
\text { solution } \mathrm{y}(\mathrm{x})\end{array}$ & 3-step block method \\
\hline 0.1 & 0.914829082 & 0.914829081 & 7.24352E-10 \\
0.2 & 0.858597242 & 0.858597238 & $3.83983 \mathrm{E}-09$ \\
0.3 & 0.830141192 & 0.830141183 & $9.324 \mathrm{E}-09$ \\
0.4 & 0.828175302 & 0.828175285 & $1.69587 \mathrm{E}-08$ \\
0.5 & 0.851278729 & 0.851278703 & $2.60999 \mathrm{E}-08$ \\
0.6 & 0.897881200 & 0.897881164 & $3.55095 \mathrm{E}-08$ \\
0.7 & 0.966247293 & 0.966247247 & $4.51295 \mathrm{E}-08$ \\
0.8 & 1.054459072 & 1.054459017 & $5.45075 \mathrm{E}-08$ \\
0.9 & 1.160396889 & 1.160396826 & $6.28431 \mathrm{E}-08$ \\
1.0 & 1.281718172 & 1.281718102 & $6.9541 \mathrm{E}-08$ \\
1.1 & 1.415833976 & 1.415833903 & $7.30536 \mathrm{E}-08$ \\
1.2 & 1.559883077 & 1.559883005 & $7.22635 \mathrm{E}-08$ \\
\hline
\end{tabular}

\section{CONCLUSION}

A block multistep method developed in this study was applied as simultaneous integrator to initial value problems. This implementation strategy was more accurate and efficient than effect the conventional (step by step) integration procedures ${ }^{[1]}$. However, predictorcorrector method can handle the categories of general third order problems in which $y^{\prime}, y^{\prime \prime}$ are present.

\section{REFERENCES}

1. Awoyemi, D.O., 2003. A P-stable linear multistep method for solving general third order ordinary differential equations. Int. J. Comput. Math., 8: 985-991. DOI: 10.1080/0020716031000079572

2. Awoyemi, D. and O. Idowu, 2005. A class hybrid collocation methods for third order of order ordinary differential equations. Int. J. Comput. Math., $\quad 82: \quad 1287-1293 . \quad$ DOI: 10.1080/00207160500112902.

3. Fatunla, S.O., 1994. A class of block methods for second order IVPs. Int. J. Comput. Math., 55: 119-133. DOI: $10.1080 / 00207169508804368$

4. Lambert, J.D., 1973. Computational Methods in Ordinary Differential Equations. John Willey and Sons, New York, USA., ISBN: 10: 0471511943, pp: 294.

5. Adee, S.O., P. Onumanyi, U.W. Sirisena and Y.A. Yahaya, 2005. Note on starting numerov method more accurately by a hybrid formula of order four for an initial value problem. J. Computat. Applied Math., 175: 369-373. DOI: 10.1016/j.cam.2004.06.016 